ARTICLE

\title{
Exhaustion of nucleus pulposus progenitor cells with ageing and degeneration of the intervertebral disc
}

Daisuke Sakai ${ }^{1,2,3, \star}$, Yoshihiko Nakamura², Tomoko Nakai1,2, Taishi Mishima², Shunichi Kato², Sibylle Grad ${ }^{4, *}$, Mauro Alini ${ }^{4, *}$, Makarand V. Risbud ${ }^{5}$, Danny Chan ${ }^{6, *}$, Kathryn S.E. Cheah $^{6}$, Ken-ichi Yamamura ${ }^{7}$,

Koichi Masuda ${ }^{8}$, Hideyuki Okano ${ }^{9}$, Kiyoshi Ando ${ }^{2,3}$ \& Joji Mochida, ${ }^{1,2,3}$

Despite the high prevalence of intervertebral disc disease, little is known about changes in intervertebral disc cells and their regenerative potential with ageing and intervertebral disc degeneration. Here we identify populations of progenitor cells that are Tie2 positive $\left(\mathrm{Tie} 2^{+}\right.$) and disialoganglioside 2 positive $\left(\mathrm{GD}^{+}{ }^{+}\right.$), in the nucleus pulposus from mice and humans. These cells form spheroid colonies that express type II collagen and aggrecan. They are clonally multipotent and differentiated into mesenchymal lineages and induced reorganization of nucleus pulposus tissue when transplanted into non-obese diabetic/severe combined immunodeficient mice. The frequency of $\mathrm{Tie}^{+}$cells in tissues from patients decreases markedly with age and degeneration of the intervertebral disc, suggesting exhaustion of their capacity for regeneration. However, progenitor cells $\left(\mathrm{Tie} 2^{+} \mathrm{GD} 2^{+}\right.$) can be induced from their precursor cells ( $\mathrm{Tie}^{+} \mathrm{GD}^{-}$) under simple culture conditions. Moreover, angiopoietin-1, a ligand of Tie2, is crucial for the survival of nucleus pulposus cells. Our results offer insights for regenerative therapy and a new diagnostic standard.

\footnotetext{
${ }^{1}$ Department of Orthopaedic Surgery, Surgical Science, Tokai University School of Medicine, Isehara, Kanagawa 259-1193, Japan. ${ }^{2}$ Research Center for Regenerative Medicine and Cancer Stem Cell, Tokai University School of Medicine, Isehara, Kanagawa 259-1193, Japan. ${ }^{3}$ The Institute of Medical Sciences, Tokai University, Isehara, Kanagawa 259-1193, Japan. ${ }^{4}$ AO Research Institute, 7270, Davos, Switzerland. ${ }^{5}$ Department of Orthopaedic Surgery and Graduate Program in Tissue Engineering and Regenerative Medicine, Thomas Jefferson University College of Medicine, Philadelphia, Pennsylvania 19107, USA.

${ }^{6}$ Department of Biochemistry, The University of Hong Kong, Hong Kong, China. ${ }^{7}$ Division of Developmental Genetics, Institute of Molecular Embryology and Genetics, Kumamoto University, Kumamoto 860-0811, Japan. ${ }^{8}$ Department of Orthopaedic Surgery, University of California, San Diego, La Jolla, California 90293-0863, USA. ${ }^{9}$ Department of Physiology, Keio University School of Medicine, Tokyo 160-8582, Japan. Correspondence and requests for materials should be addressed to D.S. (email: daisakai@is.icc.u-tokai.ac.jp).

${ }^{*}$ AO Spine Research Network
} 
$\mathrm{D}$ egeneration of the intervertebral disc (IVD) is a significant cause of back pain and has a large economic burden ${ }^{1}$. By creating instability, IVD degeneration is also a trigger for most spinal diseases and can lead to secondary spinal deformity and spinal canal stenosis ${ }^{2}$.

The IVD is composed of an inner nucleus pulposus (NP) surrounded by the annulus fibrosus (AF) and thin hyaline cartilaginous end-plates between the IVD and the adjacent vertebral bodies. The gelatinous $\mathrm{NP}$ is an avascular tissue containing extracellular matrix (ECM) comprising highly hydrated proteoglycan and collagen ${ }^{3}$. Although loss of ECMproducing cells accounts for IVD degeneration ${ }^{4}$, the pathogenesis of IVD degeneration is largely unknown and there are no effective therapies.

The IVD undergoes degenerative changes earlier in life than do other tissues of major organ systems that comprise long-lived cells that are normally replaced infrequently ${ }^{5-7}$. The functional ability of IVD cells changes over time during the process of degeneration and ageing. The cells respond to the changes induced by environmental damage and stress by entering a state of cell senescence ${ }^{8}$, during which the ECM surrounding such cells and neighbouring cells may be altered. Under these circumstances, the resilience of the IVD cell population might be ensured if the relevant stem cell populations could give rise to differentiated progeny over a person's lifetime. However, apart from a heterogeneous cell population derived from degenerate human IVD, which can differentiate into mesenchymal lineages ${ }^{9-11}$, no single-cell progenitors have been identified in NP tissue.

To identify NP stem and progenitor cells, we began with a colony-forming assay (CFA) using methylcellulose semi-solid medium, which was established to evaluate haematopoietic or endothelial stem/progenitor cells ${ }^{12,13}$ and has been used to identify tissue-specific stem/progenitor cells from various organs ${ }^{14,15}$. We sorted human and mouse NP cells using various surface markers and then scored their ability to form colonies. Following this prescreening, cells were evaluated for clonogenicity in vitro and for multipotency and self-renewal ability in vivo. Finally, we identified progenitor cells in populations purified from adult mouse $(\mathrm{m})$ and human $(\mathrm{h}) \mathrm{NP}$ cells using two markers: the tyrosine kinase receptor Tie 2 and disialoganglioside 2 (GD2). Our findings demonstrate an important role for angiopoietin-1, a ligand of Tie2, in maintaining these progenitor cells and protecting NP cells from apoptosis. The present work was undertaken to explore the hypothesis that the frequency of these progenitor cells in hNP tissues correlates with the clinical extent of IVD degeneration and ageing.

\section{Results}

Surface markers to select putative progenitor cells. To find clues to the identity of the progenitor cells in the $\mathrm{mNP}$, we evaluated NP cells from tail IVDs of 8- to 10 -week-old C57BL/6 mice in CFA in methylcellulose medium. After 10 days, adhesive fibroblastic colonies and spheroid colonies were observed. We named these two types of colonies mNP colony-forming units-fibroblastic (mNP-CFU-F) and mNP colony-forming units-spherical (mNP-CFU-S), respectively. Both types of colonies were retrieved from the medium without enzymatic digestion and were visualized with immunocytochemical staining. mNP-CFU-S were strongly positive for type II collagen and aggrecan, whereas mNPCFU-F were predominantly negative. Therefore, mNP-CFU-S were considered to develop a more NP-like phenotype than did mNP-CFU-F (Fig. 1a).

We then used a wide panel of antibodies to identify which cell surface markers correlated with the capability to form
mNP-CFU-S (Methods). GD2 was identified as the most relevant marker. This disialoganglioside is a component of the plasma membrane found mainly in the nervous system and was identified recently as a marker for bone marrow mesenchymal stem cells $(\mathrm{BM}-\mathrm{MSC})^{16,17}$ and umbilical cord mesenchymal stem cells $^{17}$. As a number of GD2-expressing cells were also positive for $\mathrm{CD} 24$, we sorted $\mathrm{GD} 2{ }^{+} \mathrm{CD} 24^{+} \mathrm{mNP}$ cells (Supplementary Fig. S1) and confirmed that these cells generated mainly mNPCFU-S, when seeded in the methylcellulose medium; the frequency increased from $19.6 \pm 2.8 / 1,000$ in unsorted cells to $103.1 \pm 13.8 / 1,000$ in sorted cells (Fig. 1b). GD2 positivity was also an indicator of proliferative capability on the plastic plates in ordinary liquid cultures (Supplementary Fig. S1) because the $\mathrm{GD} 2^{-} \mathrm{CD} 24^{+}$population showed low growth and low capacity for mNP-CFU-S formation (Supplementary Fig. S1, Fig. 1b).

To find the precursor cells of these GD2 ${ }^{+}$cells, we investigated mouse primary cultures and identified a population expressing Tie2 that comprised $\mathrm{Tie}^{+}{ }^{+} \mathrm{GD}_{2}{ }^{-}$and $\mathrm{Tie}^{+}{ }_{\mathrm{GD}}{ }^{+}$ subpopulations. To understand the role of $\mathrm{Tie}^{+}$cells, we sorted Tie $2{ }^{+} \mathrm{GD}^{-}$cells from day- 10 cultured $\mathrm{mNP}$ cells and passaged them. After 10 days, $8.0 \pm 2.7 \%$ of the cells were Tie2 ${ }^{+} \mathrm{GD} 2{ }^{+}$(Supplementary Fig. S2). Thus, $\mathrm{Tie}^{+}{ }^{+} \mathrm{GD} 2{ }^{-}$cells were confirmed to be the precursor cells of $\mathrm{Tie}^{+}{ }^{+} \mathrm{GD} 2{ }^{+}$cells. Expansion of the cultures increased the percentage of $\mathrm{Tie} 2{ }^{+} \mathrm{GD} 2{ }^{+}$cells, which then disappeared by day 30 , whereas the percentages of $\mathrm{GD}_{2}{ }^{+} \mathrm{CD} 24^{+}$and $\mathrm{GD}_{2}{ }^{-} \mathrm{CD} 24^{+}$cells increased (Supplementary Fig. S3).

In human NP cells, similar changes in the expression of Tie2, GD2 and CD24 were observed (Supplementary Fig. S4). Sorted $\mathrm{Tie}^{+} \mathrm{GD}^{-}$cells from primary hNP cells generated $\mathrm{Tie} 2{ }^{+} \mathrm{GD} 2{ }^{+}$cells; on day 14 , the $\mathrm{Tie} 2{ }^{+} \mathrm{GD} 2{ }^{+}$cell frequency was $14.5 \pm 5.6 \%$. By contrast, sorted $\mathrm{Tie} 2^{-} \mathrm{GD} 2{ }^{+}$cells generated neither Tie $2{ }^{+} \mathrm{GD} 2{ }^{-}$nor Tie $2{ }^{+} \mathrm{GD} 2{ }^{+}$cells (Supplementary Fig. S5). These changes in the markers over time suggested a hierarchy originating from $\mathrm{Tie} 2^{+} \mathrm{GD} 2^{-} \mathrm{CD} 24^{-}$cells and followed in order by $\mathrm{Tie} 2{ }^{+} \mathrm{GD} 2{ }^{+} \mathrm{CD} 24^{-}$cells, $\mathrm{Tie}^{-}$ $\mathrm{GD} 2{ }^{+} \mathrm{CD} 24^{-}$cells and $\mathrm{Tie}^{-} \mathrm{GD} 2^{-} \mathrm{CD} 24^{+}$cells. To simplify the nomenclature, we henceforth refer to the hNP cell populations as follows: $\mathrm{Tie} 2{ }^{+} \mathrm{GD} 2^{-} \mathrm{CD} 24^{-}$(Tie2 single-positive cells) as $\mathrm{T} / \mathrm{sp}$ cells, $\mathrm{Tie} 2{ }^{+} \mathrm{GD} 2{ }^{+} \mathrm{CD} 24^{-}$(Tie2 and $\mathrm{GD} 2$ double-positive cells) as TG/dp cells, Tie2 ${ }^{-} \mathrm{GD} 2{ }^{+} \mathrm{CD} 24^{-}$(GD2 single-positive cells) as $\mathrm{G} / \mathrm{sp}$ cells and $\mathrm{Tie} 2^{-} \mathrm{GD} 2^{-} \mathrm{CD} 24^{+}(\mathrm{CD} 24$ single-positive cells) as $24 /$ sp cells.

To confirm the markers for purification of hNP progenitor cells, the following experiments were performed. First, we subjected TG/dp, G/sp and 24/sp cells to the CFA. As expected, TG/dp cells had the highest frequency of hNP-CFU-S formation $(202.0 \pm 12.1 / 1000)$ among the three populations (G/sp: $120.0 \pm 14.3 / 1000,24 /$ sp: $11.4 \pm 2.8 / 1000$ ) (Fig. 1c). Consistent with the results in the mouse, immunocytochemistry showed that hNP-CFU-S were positive for type II collagen and aggrecan, in marked contrast to hNP-CFU-F (Fig. 1d). ECM production was analysed quantitatively by immunofluorescence and flow cytometry (Supplementary Methods). The percentages of cells positive for collagen and aggrecan were higher in hNP-CFU-S (type II collagen: $34.3 \pm 6.9 \%$, aggrecan: $82.4 \pm 4.9 \%$ ) than in hNP-CFU-F (type II collagen: $0.9 \pm 0.1 \%$, aggrecan: $12.1 \pm 4.9 \%$ ) (Fig. 1e). Analysis of other stem cell markers indicated that CD271 expression was higher in T/sp and TG/dp cells (> 75\% (addition of two populations expressing Tie2)) than in G/sp $(<10 \%)$ or $24 / \mathrm{sp}$ (trace) cells. CD271 has been reported as a specific marker for human MSCs of diverse origin: from bone marrow $^{18,19}$, adipose tissue ${ }^{20}$ and synovial membranes ${ }^{21}$. Expression of Flt-1 was detected only in the T/sp and TG/dp cells. However, all populations were positive for CD44, CD49f, CD56, CD73, CD90, CD105 and CD166 (Supplementary Fig. S6). 

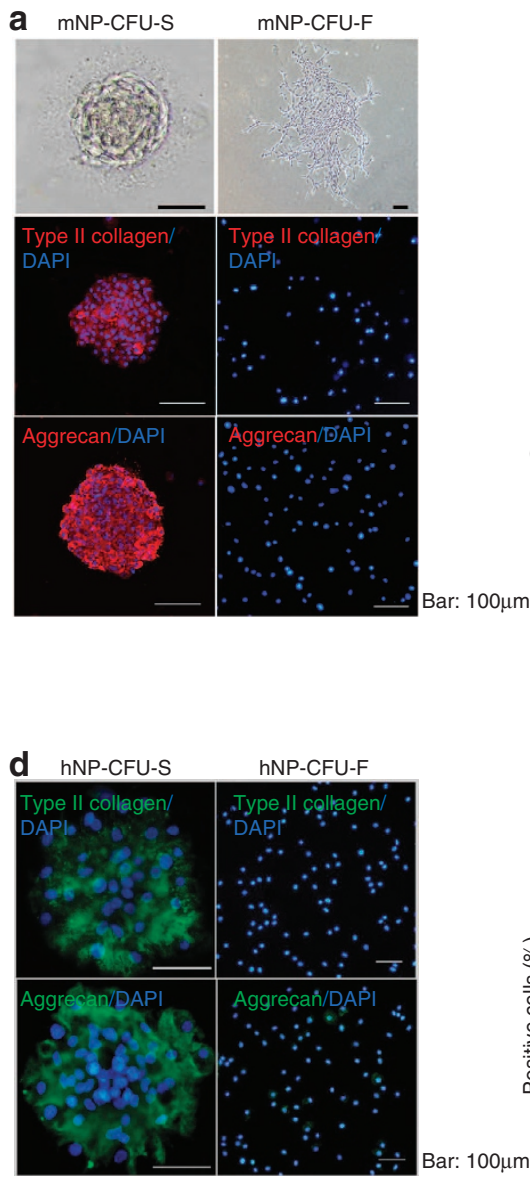
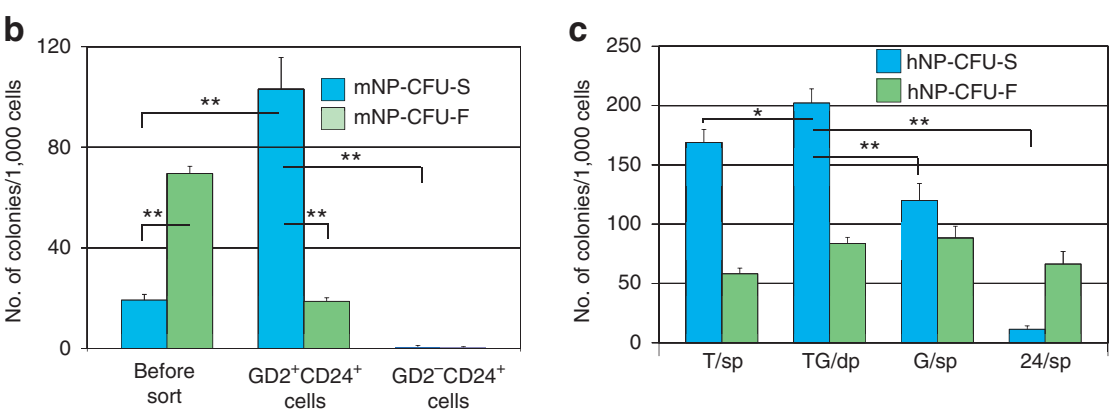

e
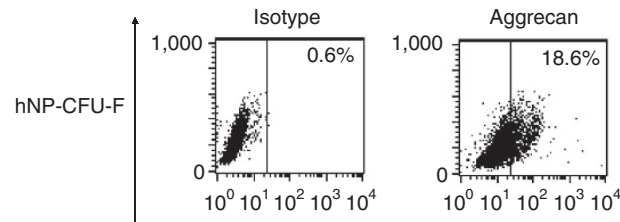

Type II collagen
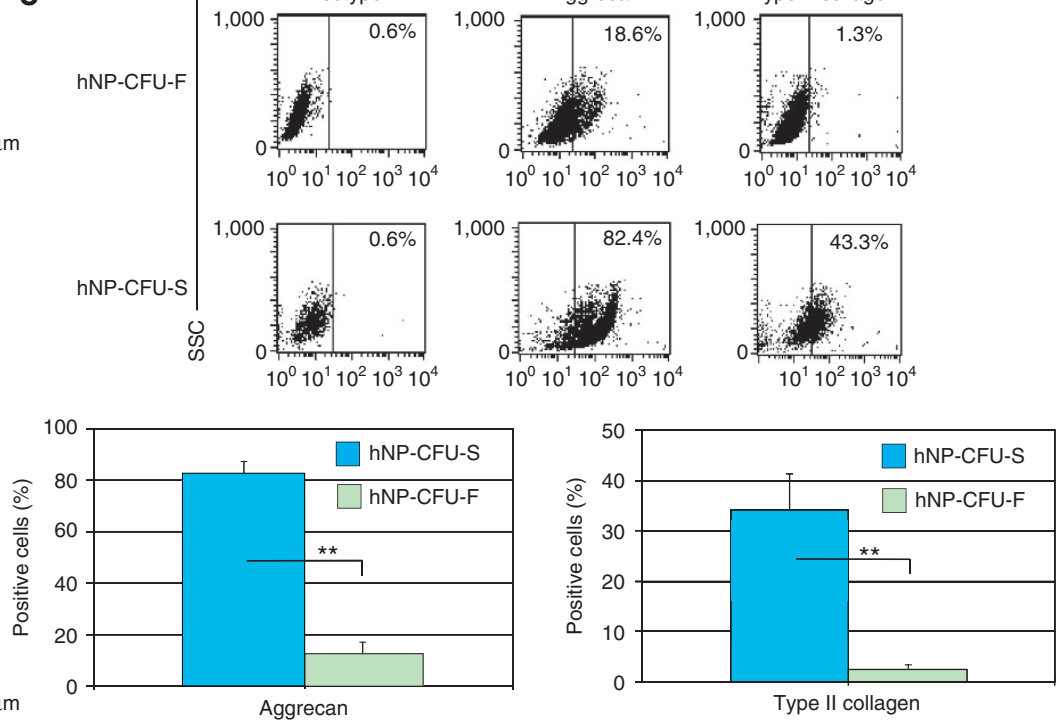

Figure 1 | Surface markers to select putative progenitor cells. (a) Two types of colonies derived from mouse primary NP cells in methylcellulose medium at 10 days (top panels): mNP-CFU-S (left) and mouse mNP-CFU-F (right). Immunocytochemical staining for the ECM indicates that mNP-CFU-S are robustly positive for type II collagen and aggrecan, whereas mNP-CFU-F are almost completely negative. Colonies were retrieved using 2 mM EDTA without enzymatic digestion and attached to slides using the Cytospin method. Scale bars, $100 \mu \mathrm{m}$. (b) One thousand mNP cells from each sorted population and unsorted cells were subjected to a CFA for 10 days. The frequency of mNP-CFU-S was higher in GD2 ${ }^{+}$sorted cells than in unsorted mNP cells. $n=5$, ${ }^{\star \star} P P 0.01$ (ANOVA with Mann-Whitney U-test). (c) One thousand human NP (hNP) cells from each sorted population were subjected to CFA for 10 days. The populations were Tie2 ${ }^{+} \mathrm{GD}^{-} \mathrm{CD} 24^{-}$(Tie2 single-positive (T/sp) cells), Tie2 ${ }^{+} \mathrm{GD} 2{ }^{+} \mathrm{CD}^{-} 4^{-}$(Tie2 and GD2 double-positive (TG/dp) cells), Tie2 ${ }^{-}$ GD2 ${ }^{+}$CD24- (GD2 single-positive (G/sp) cells) and Tie2 ${ }^{-}$GD2-CD24 ${ }^{+}$(CD24 single-positive (24/sp) cells). TG/dp cells had the greatest hNP-CFU-S formation among the four populations. $n=5,{ }^{\star} P<0.05$, ${ }^{\star} P<0.01$ (ANOVA with Mann-Whitney U-test). (d) Comparison of ECM production between hNP-CFU-S and hNP-CFU-F. hNP-CFU-S were immunopositive for type II collagen and aggrecan, in contrast to the low expression in hNP-CFU-F. Scale bars, $50 \mu \mathrm{m}$. (e) Quantitative comparison of ECM production between hNP-CFU-S and hNP-CFU-F by flow cytometry. The cytoplasm of enzymatically dispersed and membrane-permeabilized cells from the colonies was stained. A higher percentage of immunopositive cells was detected in the cells from hNP-CFU-S than in those from hNP-CFU-F. $n=3,{ }^{\star \star} P<0.01$ (ANOVA with Mann-Whitney U-test). Data are represented as mean \pm s.d. DAPI, 4',6-diamidino-2phenylindole.

These results support our proposed cellular hierarchy led by $\mathrm{T} / \mathrm{sp}$ cells because T/sp and TG/dp cells were distinct from the two downstream G/sp and 24/sp populations; these results also confirm Tie2 and GD2 as markers. To assess whether harvested NP cells include migrated macrophage or cells from penetrated vasculature, we used flow cytometry to investigate the expression of markers of macrophages (CD45, CD14, CD11c), endothelial cells (CD31, CD34, CD144, Ulex europaeus Lectin, Von Willebrand Factor) and pericytes (CD146). We confirmed the lack of contamination by cells of haematopoietic or endothelial origin (Supplementary Fig. S7), which was probably due to the precautions taken during sample collection (Methods).

Single-cell-derived multipotent cells in TG/dp cells. We predicted that progenitor cells were present within the population expressing Tie 2 and performed the following experiments to verify their stem cell properties. However, because single T/sp cells did not proliferate enough for the study, TG/dp were used for clonal experiments. To demonstrate that hNP-CFU-S were derived from single cells, we inoculated single TG/dp cells separately into methylcellulose medium and confirmed the generation of hNP-CFU-S (Fig. 2a).

The results of population-doubling assays started from single TG/dp or single 24/sp cells showed that the TG/dp clones had higher proliferative potential that expanded greatly beyond 27 cell doublings, whereas the $24 / \mathrm{sp}$ clones were markedly inferior (Fig. 2b).

To evaluate their multipotent differentiation capability in vitro, TG/dp clonal cultures were subjected to 21 days of induction towards adipo-, osteo- or chondrogenesis (Fig. 2c). Nine of the 10 clones tested were bipotent (that is, could differentiate into two cell lineages) and four were tripotent (could differentiate into all three cell lineages). Thus, these clones were multipotent (Table 1). 
a $\quad$ A single $T G / d p$

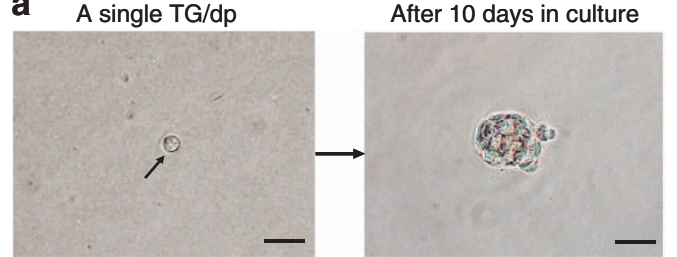

b

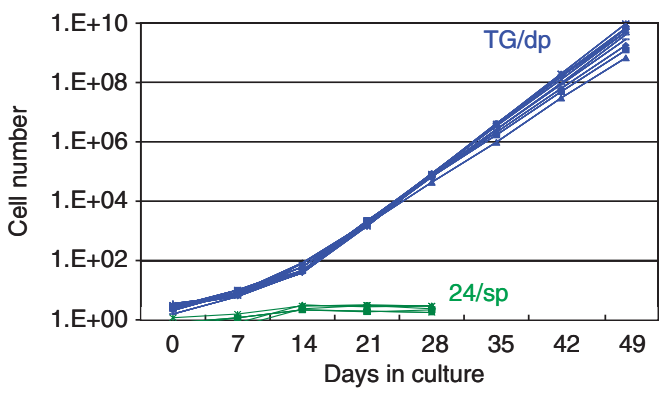

C

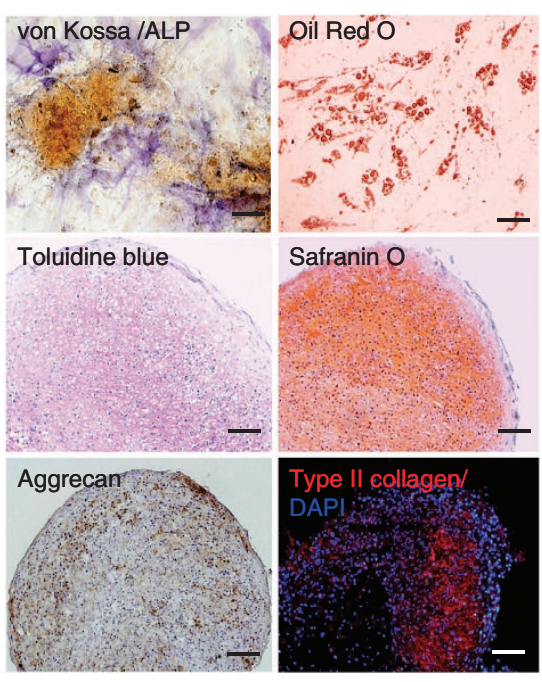

d

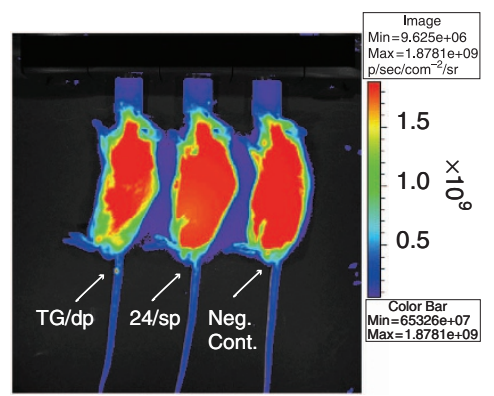

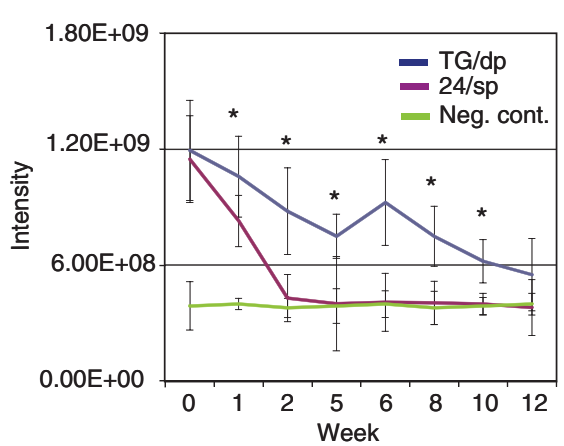

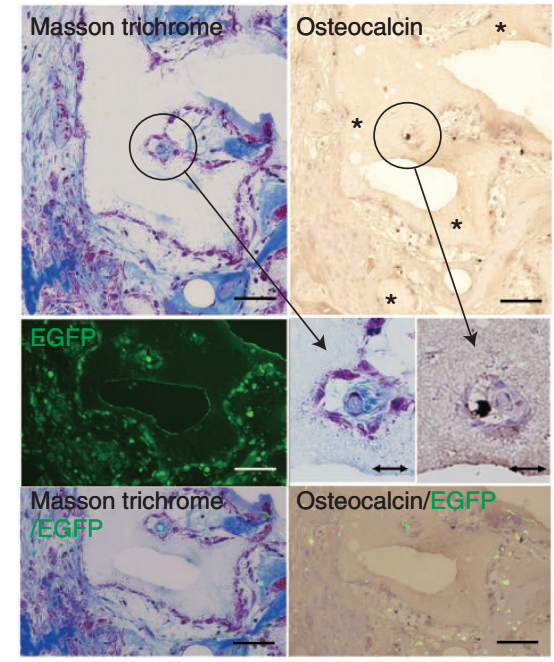

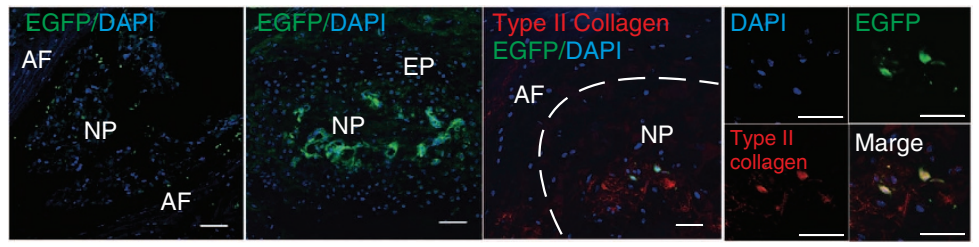

Figure 2 | Single-cell-derived multipotent cells in TG/dp cells. (a) Single TG/dp cells were purified from 7-day cultured hNP cells and inoculated into methylcellulose medium in 96-well culture plates. Single-cell-derived hNP-CFU-S were observed on day 10. (b) Population-doubling assays of TG/dp and 24/sp clones. TG/dp clones proliferated beyond 27 doublings, whereas 24/sp clones did not. (c) Clonal differentiation assays for mesenchymal lineages. Expanded clonal cultures from single TG/dp cells were cultured with induction medium for 21 days, and osteogenesis (von Kossa/ALP: Alkaline phosphatase), adipogenesis (Oil Red O) and chondrogenesis (toluidine blue, Safranin O, aggrecan and type II collagen) were detected. Scale bars, $100 \mu \mathrm{m}$. (d) Survival of the transplanted cells in vivo detected by bioluminescence imaging (BLI). An EGFP-labelled TG/dp clone or EGFP-labelled 24/sp clone was injected into injured tail IVDs of NOD/SCID mice $\left(1 \times 10^{5} / \mathrm{IVD}\right.$, respectively). BLI shows the survival of the TG/dp clone at day 31 , whereas the $24 /$ sp clone disappeared (upper left). Negative control mice without cell transplantation were used for calibration of autofluorescence. Superior survival of TG/dp cells is shown in the graph, $n=3,{ }^{\star} P<0.05$, (ANOVA with Tukey's post hoc test). EGFP-labelled TG/dp clone cells were detected inside the harvested disc sections (bottom left, mid-coronal; middle two panels, transverse sections). Coexpression of type II collagen with EGFP was detected by immunostaining (low magnification and high magnification, in three colours). Scale bars, $50 \mu \mathrm{m}$. (e) For osteogenesis in vivo, an EGFP-labelled TG/dp clone $\left(1 \times 10^{5}\right)$ was injected with a hydroxyapatite scaffold into a femoral bone defect model in NOD/SCID mice. Eight weeks later, Masson's trichrome staining (top left) of the paraffin section identified blue collagenous fibres surrounding the EGFP + cells (bottom left). Immunohistochemical staining showed positive staining for osteocalcin (top right) and osteocalcin expression in the EGFP ${ }^{+}$cells (bottom right) in a cavity within the hydroxyapatite $\left(^{\star}\right)$. Straight bars, $50 \mu \mathrm{m}$; arrowed bars, $20 \mu \mathrm{m}$, data are represented as mean \pm s.d. DAPI, 4',6-diamidino-2-phenylindole; Neg. cont., negative control.

To evaluate their potential in vivo, differentiation towards the NP and osteogenesis was assessed. Briefly, a TG/dp clone was transduced with the enhanced green fluorescent protein (EGFP) gene, and the cells were injected into injured tail IVDs of nonobese diabetic/severe combined immunodeficient (NOD/SCID) mice $\left(1 \times 10^{5} / \mathrm{IVD}\right)$. As a sham control, the same number $\left(1 \times 10^{5} / \mathrm{IVD}\right)$ of cells from a $24 / \mathrm{sp}$ clone was injected.
Bioluminescence imaging was used to assess the survival and residence time of the implanted clones. Twelve weeks later, histological analysis of the harvested TG/dp clone-injected IVDs showed survival of $\mathrm{EGFP}^{+}$cells with an NP phenotype as detected by type II collagen coexpression, whereas the cells disappeared within 1-2 weeks in the sham control (Fig. 2d). To evaluate osteogenesis, an EGFP-labelled TG/dp clone $\left(1 \times 10^{5}\right)$ 


\begin{tabular}{|c|c|c|c|c|}
\hline Clone ID & Chon. & Adipo. & Osteo. & Multipotent \\
\hline 1 & - & + & + & $\star \star$ \\
\hline 2 & + & + & + & $\star \star \star \star$ \\
\hline 5 & - & + & + & $\star \star$ \\
\hline 7 & + & - & - & * \\
\hline 10 & - & + & + & $\star \star$ \\
\hline 14 & + & + & + & $\star \star \star \star$ \\
\hline 15 & + & + & + & 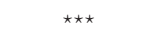 \\
\hline 17 & - & + & + & $\star \star$ \\
\hline 18 & - & + & + & $\star \star$ \\
\hline 20 & + & + & + & 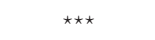 \\
\hline Ratio & $5 / 10$ & $9 / 10$ & $9 / 10$ & $\begin{array}{l}\text { Tri-: 4/10 } \\
\text { Bi-: } 9 / 10\end{array}$ \\
\hline $\begin{array}{l}\text { Results from } \\
\text { bipotent clon } \\
\text { *Unipotent, * }\end{array}$ & $\begin{array}{l}\text { erentiation } \\
{ }^{\star \star \star} \text { Tripote }\end{array}$ & $s$ for three & chymal line & howed tri- and \\
\hline
\end{tabular}

was injected together with a hydroxyapatite scaffold into a model femoral bone defect in NOD/SCID mice. Eight weeks later, healed bone was harvested and examined. Masson's trichrome staining identified blue collagenous fibres surrounding $\mathrm{EGFP}^{+}$cells, which were also osteocalcin positive, as shown by immunostaining (Fig. 2e). Thus, the implanted TG/dp clone underwent histocompatible differentiation without prior induction in vitro. These results indicate that the TG/dp clones are multipotent.

Self-renewal capacity and NP tissue reorganization. We performed serial cell transplantations to assess the abilities for selfrenewal and NP tissue reorganization of TG/dp cells. Sorted hNP cells $\left(1 \times 10^{5}\right)$ transduced with the EGFP gene were transplanted subcutaneously with lethally irradiated (15 Gy) allogeneic hNP tissue $(0.10 \mathrm{~g})$ as a scaffold into five NOD/SCID mice, and the resulting tissues were harvested after 8 weeks (Fig. 3a). G/sp and $24 /$ sp cells were used as controls. Only TG/dp achieved long-term engraftment in all recipients, and a number of labelled cells were detected in the transplants. By contrast, transplanted tissue scaffolds with or without control cells shrank markedly (Fig. 3b, Supplementary Fig. S8). Histological analysis detected robust type II collagen and proteoglycan staining in the TG/dp transplants, indicating reorganization of NP tissue by TG/dp cells (Fig. 3b). Moreover, only TG/dp cells maintained the tissue weight at $0.11 \pm 0.03 \mathrm{~g}$, in marked contrast to the decrease in the controls (Fig. 3c). Flow cytometry detected $29.0 \pm 9.3 \%$ labelled cells in the cells retrieved by enzymatic digestion from the TG/dp transplants, whereas only $4.4 \pm 3.8 \%$ were labelled in G/sp transplants and $0.5 \pm 0.4 \%$ in $24 /$ sp transplants (Fig. 3d, Supplementary Fig. S9). These results demonstrate the survival of TG/dp in vivo.

Subsequently, the retrieved $\mathrm{EGFP}^{+}$cells from the TG/dp transplants were expanded and subjected to a mesenchymal lineage differentiation assay in vitro and to secondary transplantation. After primary transplantation and expansion, TG/dp maintained their tripotency in two of five recipients, and cells from all recipients showed bipotent capability (Fig. 3e, Supplementary Table S1). Eight weeks after the secondary transplantation, $5.7 \pm 4.3 \%$ of the retrieved cells from five recipients were $\mathrm{EGFP}^{+}$and could still proliferate when passaged (Fig. 3g, Supplementary Fig. S10). Histological analysis of the harvested transplants showed that they had been repopulated with labelled cells, which were surrounded by type II collagen and proteoglycan (Fig. 3f). These results suggest the existence of NP progenitor cells in the secondarily transplanted cells. Thus, only the multipotent cells in the TG/dp population that could differentiate into the three mesenchymal and NP lineages were self-renewing and, consequently, only they survived through the long-term xenograft trial.

Function of Tie2-Ang-1 signalling in hNP. In hNP tissue, $\mathrm{Tie}^{+}$NP cells coexpressing Ang-1, a ligand of Tie2, were detected by immunohistochemistry (Fig. 4a). We detected large amounts of Ang-1 in the supernatant of the NP cell cultures by enzyme-linked immunosorbent assay (Supplementary Fig. S11). We added Tie2-blocking antibody to the serum-free culture medium and found that the number of apoptotic NP cells increased about twofold (Fig. 4b). This result suggested an innate anti-apoptotic effect of endogenous Ang-1 on hNP cells. We used real-time reverse transcription-polymerase chain reaction (RTPCR) analysis to investigate the expression of $A n g-1$ mRNA in T/ sp, TG/dp, G/sp and 24/sp cells. Gene expression in Tie2 ${ }^{-} \mathrm{GD} 2^{-}$ CD24- cells was used as a control. The expression of Ang-1 mRNA was lower in T/sp than in the other three populations (Fig. 4c), suggesting that the downstream TG/dp, G/sp and 24/sp populations supplied Ang-1 to T/sp.

We next used soluble human Ang-1 protein (sAng1) or coculture with feeder cells (murine stromal cells overexpressing human Ang-1 (AHESS-5)) ${ }^{22}$ to demonstrate Ang-1-dependent upregulation of hNP-CFU-S generation and selective enhancement of proliferation of $\mathrm{Tie}^{+}$populations (T/sp, TG/ $\mathrm{dp})$. Culture with sAng1 increased hNP-CFU-S generation $1.9 \pm 0.1$ times (Fig. 4d). Coculture with AHESS-5 increased generation of hNP-CFU-S $3.4 \pm 0.2$ times (Fig. 4e) and proliferation of $\mathrm{T} / \mathrm{sp} 3.1 \pm 0.4$ times and TG/dp $3.2 \pm 0.4$ times (Fig. 4f). All these effects were abolished completely by addition of Tie2-blocking antibody, thereby confirming the crucial role of Tie2-Ang-1 signalling in Tie ${ }^{+}$hNP cells.

Expression of Tie2 and GD2 in IVD tissues. To confirm the expression of Tie2 and GD2 in the IVD tissues, we stained mouse and human IVD tissues. Haematoxylin and eosin staining of the paraffin sections shows the histological profiles of mouse and human IVD tissues (Fig. 5a,b). Immunohistochemical staining detected both Tie2 and GD2 in cells in the NP area in mouse tail IVDs. Fixed IVD samples were frozen without decalcification and sectioned transversely. The locations of the marker molecules and the mNP cells are shown with phalloidin staining of F-actin in the cytoskeleton (Fig. 5a). We also identified these markers in frozen hNP sections. hNP cells stained positively with Tie2 or GD2 were detected clearly in the NP area in sections harvested from young donors (Fig. 5b).

Decrease of Tie2 ${ }^{+}$NP cells with ageing and degeneration. To understand the clinical relevance of Tie $2^{+}$cells (T/sp and TG/dp cells), we analysed freshly dispersed hNP cells from surgical specimens with various degrees of IVD degeneration (Supplementary Table S2). We found a large variation in the percentage of living cells that could be analysed after tissue digestion in relation to the donor's age and the extent of degeneration. Therefore, we excluded propidium iodide (PI)positive cells and analysed only the living cells using the live-gate system in flow cytometry (Methods). The frequency of Tie $2^{+}$(T/ sp and TG/dp) cells and hNP-CFU-S in hNP cells decreased with the age of the donors (Fig. $6 \mathrm{a}-\mathrm{c}$ ). The decreases in Tie ${ }^{+}(\mathrm{T} / \mathrm{sp}$ and TG/dp) cell frequency and hNP-CFU-S frequency correlated with the extent of disc degeneration (Fig. 6d-f) graded by morphology and magnetic resonance imaging. These data strongly suggest that exhaustion of the resources for regeneration may cause various changes within IVDs and support the relevance of Tie2 as a marker of NP progenitor cells. 


\section{Discussion}

We have successfully identified NP progenitor cells in the $\mathrm{Tie}^{+}$and $\mathrm{GD}_{2}{ }^{+}$populations from mouse and human IVDs. These cells were highly proliferative and were clonally capable of differentiating into multiple mesenchymal and NP lineages. Moreover, this multipotent ability was sustained through long-term engraftment and included the ability for self-renewal.
IVD cells have been characterized poorly, which hinders the development of strategies for preventing and treating IVD degeneration. Cartilage chondrocytes have often been used as a reference for investigation of IVD cells. Chondrocytes are unique among non-transformed cells in that they are capable of anchorage-independent growth in soft $\mathrm{agar}^{23}$. In the current study, we found that NP cells could form clonal spheroid colonies in methylcellulose medium, and we used this characteristic to

a

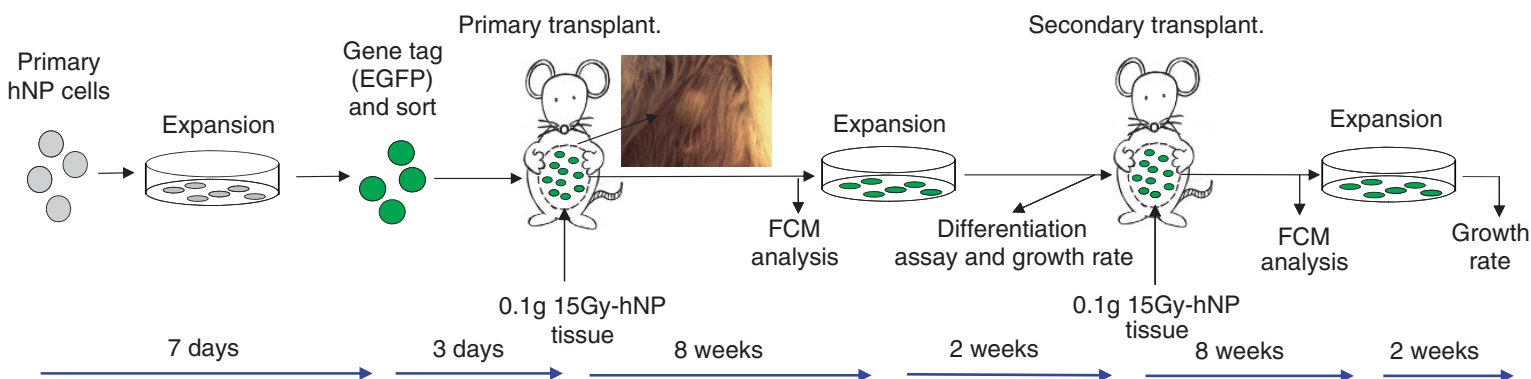

b
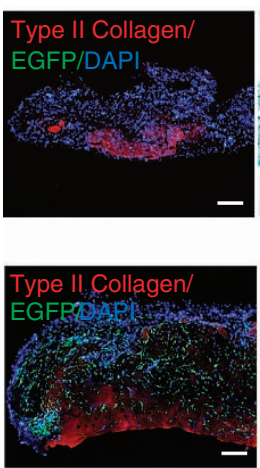

Tissue alone

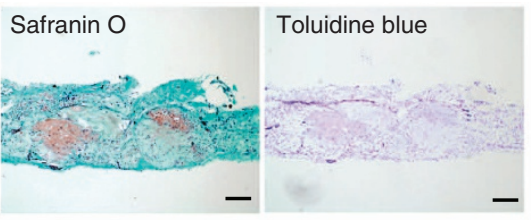

$\mathrm{TG} / \mathrm{dp}$ with tissue

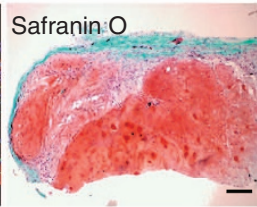

Toluidine blue

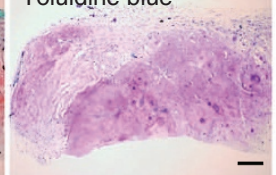

e
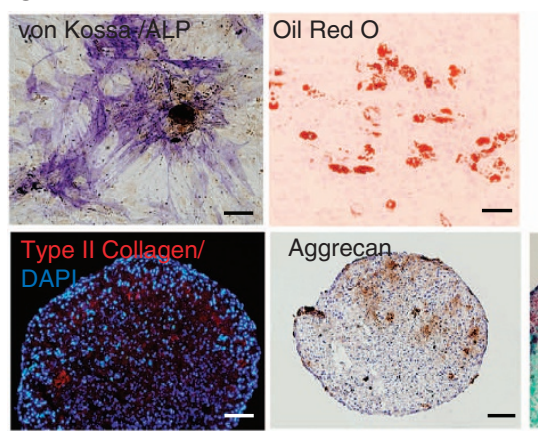

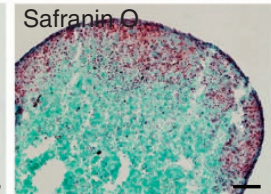

f

TG/dp with tissue

C

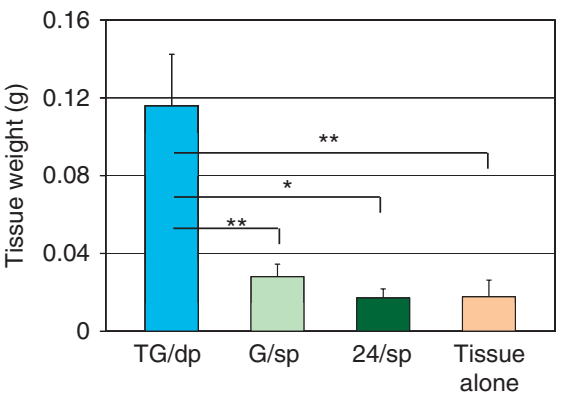

d
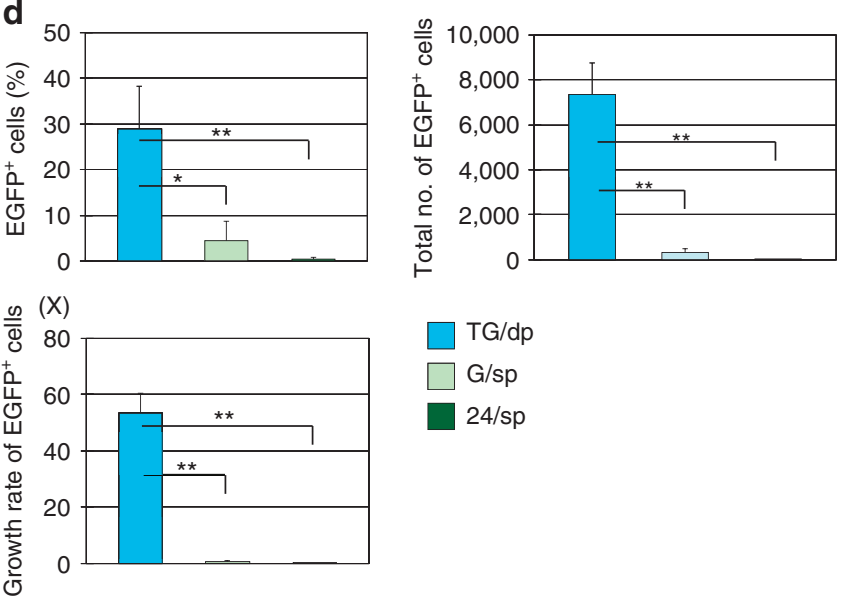

$\mathrm{TG} / \mathrm{dp}$

$\mathrm{G} / \mathrm{sp}$

$24 / \mathrm{sp}$
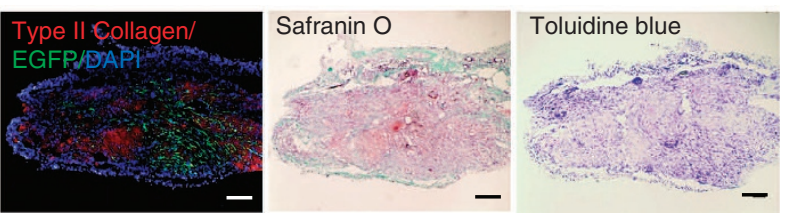

(X)
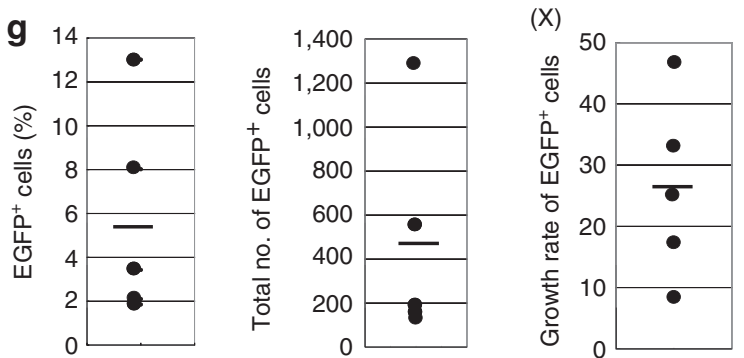
identify NP progenitor cells. We identified the recently reported BM-MSC marker GD2 $2^{16}$ as a surface marker of NP-CFU-S. Although the exact mechanism explaining the difference between fibroblastic and spherical colony-forming cells is unclear, cell sorting with GD2 provided fundamental information about mouse and human NP cells. a

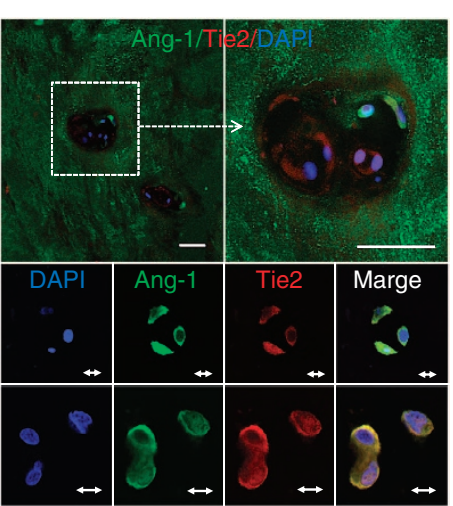

d

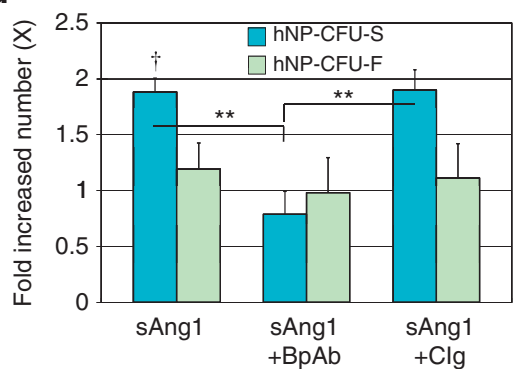

b
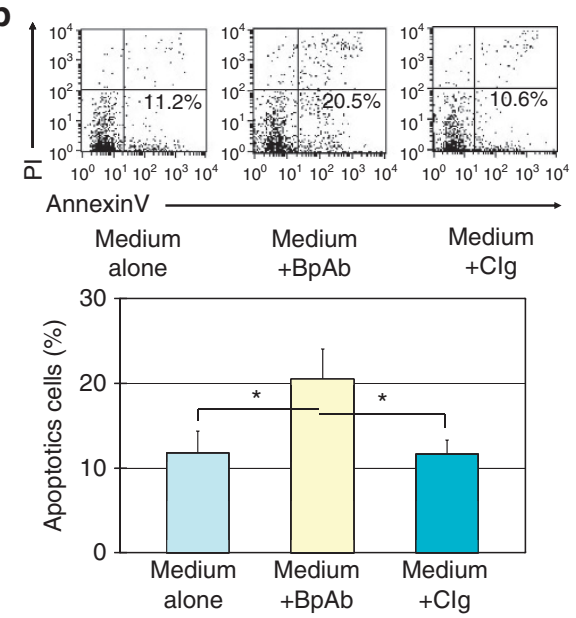

e

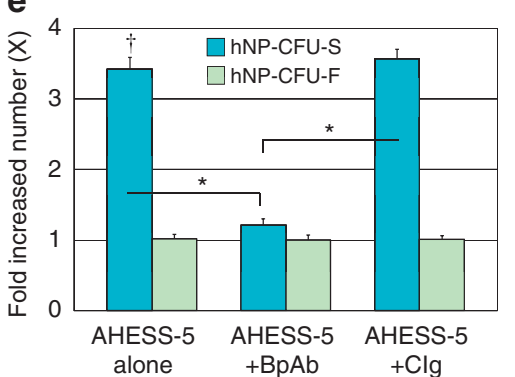

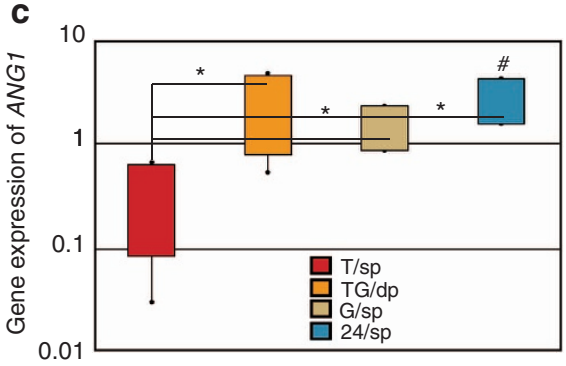

f

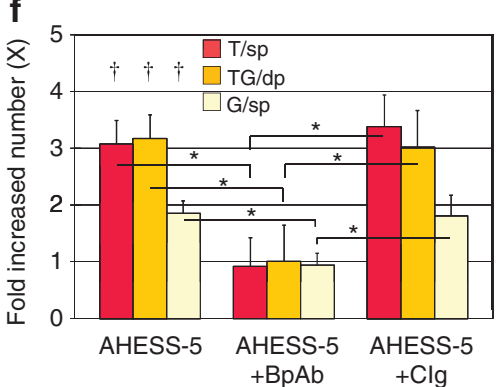

Figure 4 | Function of Tie2-Ang-1 signalling in the hNP. (a) In hNP tissues, immunohistochemistry detected Tie ${ }^{+}$cells and Ang-1 expression in the matrix and cells (top left, cavities in hNP tissue; top right, the cavity magnified). Coexpression of Tie2 and Ang-1 was also detected (middle and bottom panels). Straight bars, $50 \mu \mathrm{m}$; arrowed bars, $10 \mu \mathrm{m}$. (b) Apoptosis assay in a serum-free culture of hNP cells. Addition of Tie2-blocking polyclonal antibody (BpAb) increased the number of apoptotic hNP cells after $48 \mathrm{~h}$ compared with medium alone or addition of goat control lg (Clg). Apoptotic cells were identified by flow cytometry as Annexin V-positive and PI-negative cells. $n=3,{ }^{\star} P<0.05$ (ANOVA with Mann-Whitney U-test). (c) Quantitative comparison by real-time RT-PCR of Ang-1 mRNA expression in the sorted hNP cell fractions. Gene expression levels were normalized to $18 \mathrm{~S}$ rRNA and are presented relative to the levels in Tie2-GD2-CD24- cells. $n=3$, ${ }^{\star} P<0.05$ between the sorted cell fractions, $\# P<0.05$ compared with the Tie2-GD2 ${ }^{-}$CD24- cell control (ANOVA with MannWhitney U-test). (d) sAng1 significantly upregulated hNP-CFU-S generation. Note the significant reduction in hNP-CFU-S generation with addition of Tie2 BpAb compared with the Clg. $n=5,{ }^{\star \star} P<0.01, \uparrow P<0.05$ compared with the no-sAng1 control (ANOVA with Mann-Whitney U-test). (e) Cocultures in contact with AHESS-5 feeder cells through a culture insert (murine stromal cells overexpressing human Ang-1). The frequency of hNP-CFU-S increased significantly in the NP cells cocultured with AHESS-5, whereas the frequency of hNP-CFU-F did not change. Addition of Tie2 BpAb to the coculture medium blocked this effect. $n=3$, ${ }^{\star} P<0.05, \dagger P<0.05$ compared with the HESS-5 (without overexpression of Ang-1) control (ANOVA with Mann-Whitney U-test). (f) A comparison of the three hNP cell populations (T/sp, TG/dp and G/sp) shows marked Ang-1-dependent growth in the two Tie2+ populations (T/sp and TG/dp). $n=3$, ${ }^{\star} P<0.05$ between the groups, $\uparrow P<0.05$ compared with the HESS-5 control. Data are represented as mean $\pm \mathrm{s}$.d.

Figure 3 | Self-renewal capacity and NP tissue reorganization. (a) Strategy for testing the self-renewal capacity of TG/dp cells by serial subcutaneous transplantation into NOD/SCID mice. Sorted cell populations (TG/dp, G/sp and 24/sp cells) transduced with the EGFP gene were transplanted subcutaneously with lethally irradiated ( $15 \mathrm{~Gy}$ ) allogeneic hNP tissues $(0.10 \mathrm{~g})$ as a scaffold. FCM, Flow cytometry. (b) Transplantation of scaffold tissue alone (upper panels). Transplantation of EGFP-labelled TG/dp cells $\left(1 \times 10^{5}\right)$ with tissue (lower panels). A number of EGFP+ cells were detected in the TG/dp transplanted specimen, and histological analyses detected robust type II collagen and proteoglycan staining (Safranin O and toluidine blue). Scale bars, $200 \mu \mathrm{m}$. (c) Harvested tissue weight was maintained when transplanted with TG/dp. However, tissues transplanted without cells or with other cell populations (G/sp, 24/sp cells) lost considerable weight. $n=5,{ }^{\star \star} P<0.01$ (ANOVA with Mann-Whitney U-test). (d) Percentage of EGFP ${ }^{+}$cells detected by flow cytometry in cells retrieved from the transplants: $29.0 \pm 9.3 \%$ from TG/dp transplants, $4.4 \pm 3.8 \%$ from G/sp transplants and $0.5 \pm 0.4 \%$ from $24 / \mathrm{sp}$ transplants (top left). The corresponding cell numbers are shown (top right). After retrieval, the growth rates of cells cultured for 2 weeks were compared. The growth rate was higher in TG/dp transplants than in G/sp or 24/sp transplants (bottom left). $n=5{ }^{\star} P<0.05$, ${ }^{\star \star} P<0.01$ (ANOVA with Mann-Whitney U-test). (e) EGFP ${ }^{+}$cells from the TG/dp transplants maintained their multipotent differentiation ability towards osteogenesis (von Kossa/ALP), adipogenesis (Oil Red O) and chondrogenesis (type II collagen, aggrecan and Safranin O). Scale bars, $100 \mu \mathrm{m}$. (f) The expanded EGFP + cells from the primary TG/dp transplants were subjected to secondary transplantation with lethally irradiated (15 Gy) allogeneic hNP tissue $(0.10 \mathrm{~g})$ as a scaffold. EGFP+ cells and type II collagen (left) and proteoglycan staining (Safranin $\mathrm{O}$ and toluidine blue, middle and right) were still detected in frozen sections of the harvested tissues. Scale bars, $200 \mu \mathrm{m}$. (g) The ratio and cell number of EGFP ${ }^{+}$cells retrieved from five individual recipients after secondary transplantation. EGFP-labelled TG/dp cells underwent two rounds of transplantation and still maintained high growth capacity for 2 weeks after retrieval. Data are represented as mean \pm s.d. 
a

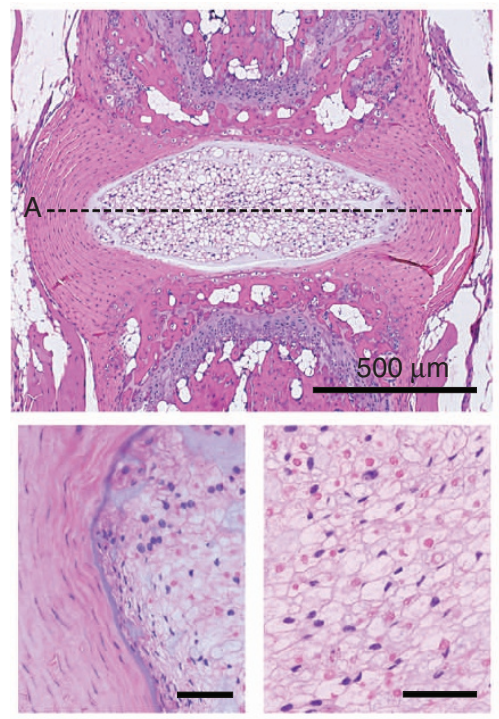

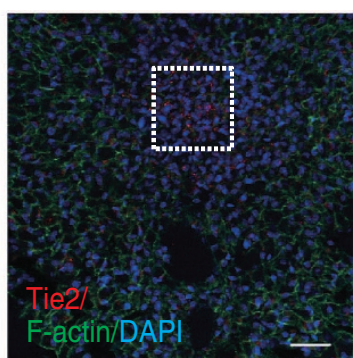
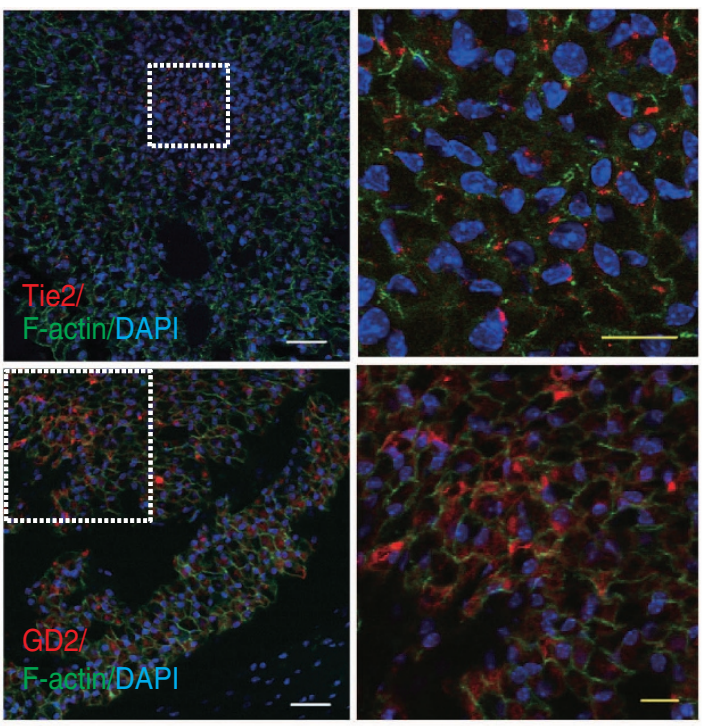

b

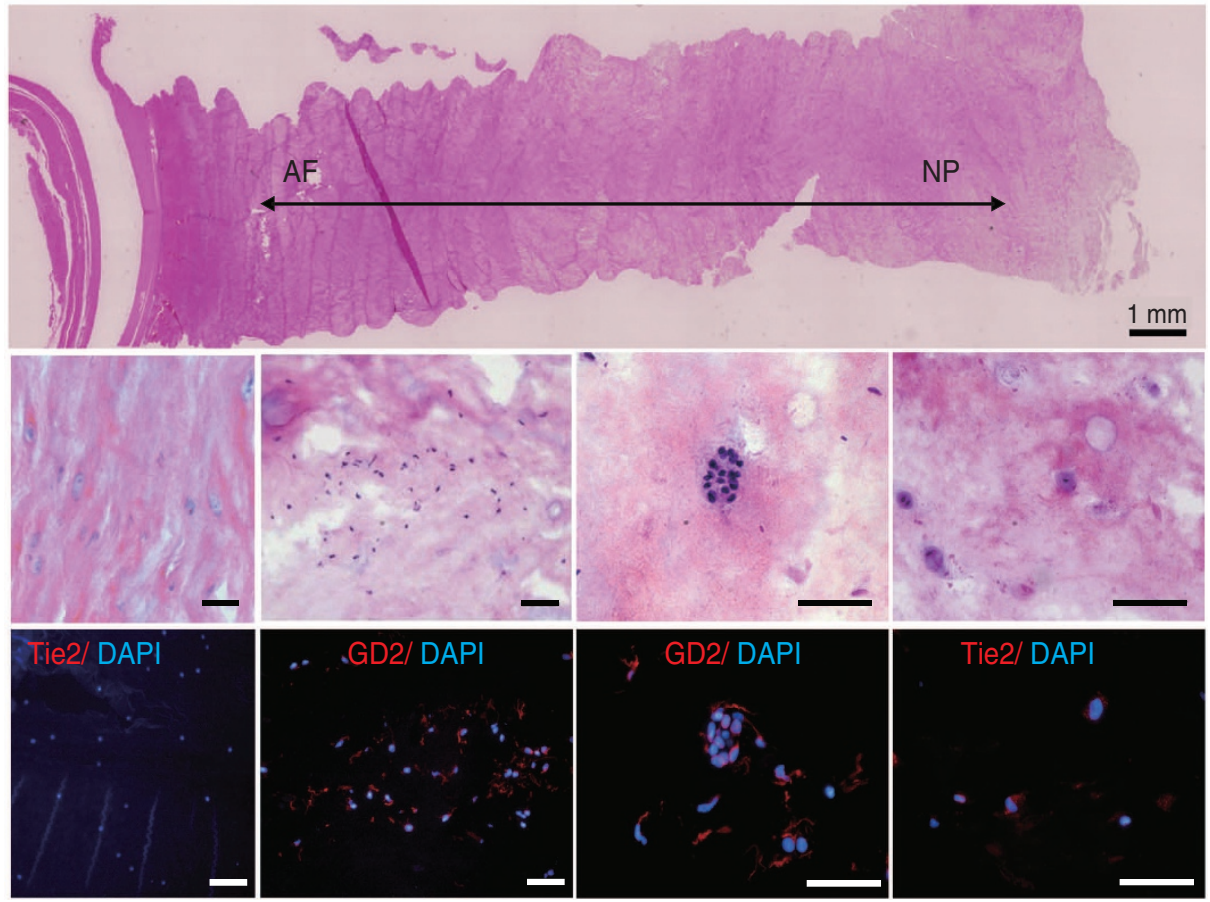

Figure 5 | Expression of Tie2 and GD2 in IVD tissues. (a) Histological analysis of paraffin sections of 9-week-old mouse tail IVDs sectioned in the midcoronal plane and stained with haematoxylin and eosin ( $\mathrm{HE}$, top left). Line A indicates the direction of the sectioning, and the location was identified in the frozen sections used for immunofluorescence. The HE image on the far left shows the AF and NP border, and the right HE image shows the central NP. Fluorescent staining results show visualization of F-actin fibres of the cytoskeleton of mNP cells by phalloidin reagent (green). Expression of Tie2 (top middle, red in mNP cells; top right, the stained cells magnified) and GD2 (bottom middle, red in mNP cells; bottom right, stained cells magnified) on the cell surface was confirmed. Yellow scale bars, $20 \mu \mathrm{m}$; black and white bars, $50 \mu \mathrm{m}$. (b) Histological paraffin section of human IVD tissue (Thompson grade II) stained with HE identifies the corresponding locations in the following frozen sections indicated in the middle and lower panels (top panel; scale bar, $1 \mathrm{~mm}$ ). Middle (HE) and bottom (immunofluorescence) panels of sections obtained from the AF (far left) to central NP (far right). No apparent immunostaining was detected for Tie2 or GD2 in the AF area (bottom left), but GD2 ${ }^{+}$and Tie2 ${ }^{+}$cells were detected (bottom centre to far right) in other areas. Scale bars, $50 \mu \mathrm{m}$. DAPI, 4',6-diamidino-2-phenylindole.

Our studies of GD2 ${ }^{+}$cells also led us to the discovery of their Tie ${ }^{+}$precursors. In addition to its expression in endothelial cells, Tie2 has been reported to be expressed in haematopoietic and neural stem cells, and is thought to regulate stem cell quiescence by promoting adhesion to the Tie $2 /$ Ang- 1 stem cell niche ${ }^{24}$. The identification of the Tie2/Ang-1 system, which is usually associated with angiogenesis in the avascular IVD microenvironment, is surprising. However, NP cells have been reported to express vascular endothelial growth factor-A (VEGFA) and its receptor, membrane-bound vascular endothelial growth factor receptor-1 (mbVEGFR-1) ${ }^{25}$. The investigators also showed that VEGF-A expression is promoted by hypoxic conditions and that this has an important role as a survival factor in the NP. As expression of Tie2/Ang- 1 can be induced by local tissue hypoxia ${ }^{26}$, it is possible that the expression of Tie2/Ang-1 is induced in NP cells. Similar to the function of 
a

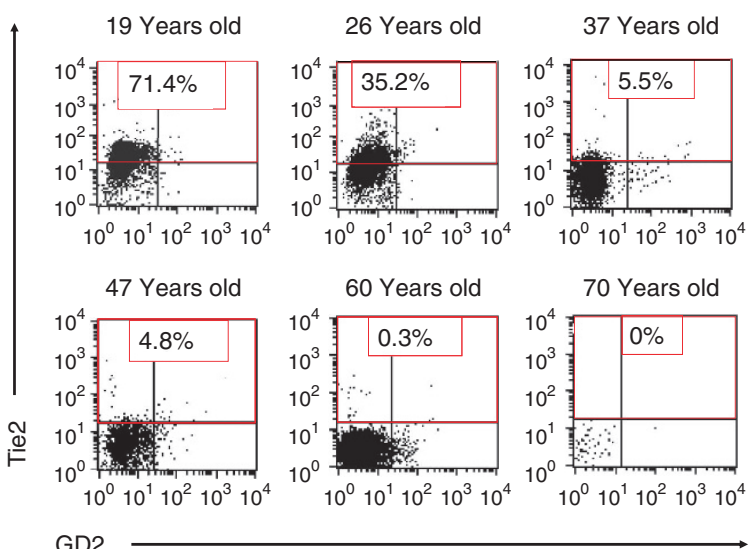

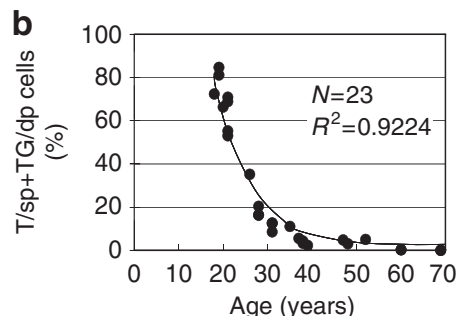

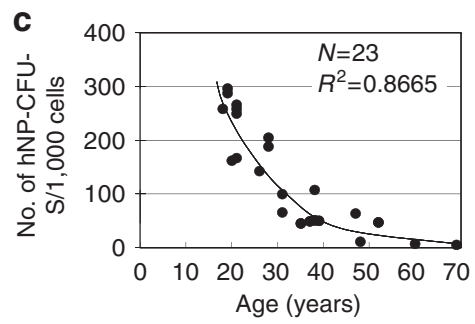

f

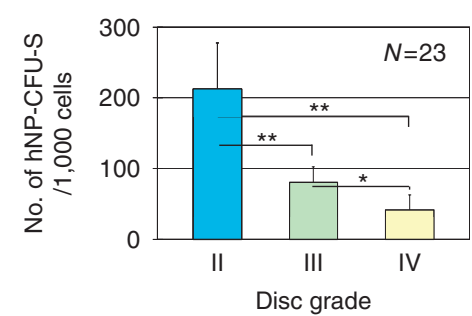

Figure 6 | Decrease of Tie2 ${ }^{+}$NP cells with ageing and degeneration. hNP cells donated by patients were studied together with their clinical profiles. Cells were freshly dispersed and only cells detected by flow cytometry within the live and the PI-negative gate were analysed. (a) Representative flow cytometry data of Tie2 and GD2 cell positivity in different age groups. (b) The frequency of Tie2 ${ }^{+}$cells (T/sp and TG/dp hNP cells) began to decrease before 20 years of age and correlated negatively with age $\left(n=23, R^{2}=0.9224\right)$. (c) The frequency of hNP-CFU-S generation also decreased with age $\left(n=23, R^{2}=0.8665\right)$. (d) The frequency of Tie2 ${ }^{+}$cells (T/sp and TG/dp hNP cells) decreased in relation to the extent of disc degeneration graded by morphology and (e) with disc degeneration graded by diagnostic magnetic resonance imaging $(n=11)$. (f) The frequency of hNP-CFU-S generation decreased in relation to the extent of disc degeneration graded by morphology $(n=23)$. ${ }^{\star} P<0.05,{ }^{\star \star} P<0.01$ (ANOVA with Mann-Whitney $U$-test). Data are represented as mean \pm s.d.

VEGF-A/mbVEGFR-1 in the resistance of the NP to apoptosis under hypoxic conditions, Tie2/Ang-1 signalling may have a role in NP cell maintenance and survival.

$\mathrm{CD} 24$ has been reported as an NP-specific marker in the rat ${ }^{27}$. We found that in mNP cells, CD24 was associated with inferior proliferation in adhesive ordinary cultures and with low colonyforming capability in methylcellulose medium. These effects contrasted with those of GD2, which correlated with NP-CFU-S frequency and high proliferative capability (Fig. 1b, Supplementary Fig. S1). In addition, Tie2 identified precursor cells that exhibited multipotency and self-renewal capacity. We also confirmed the expression of these three markers in hNP cells, which can be used to sort cells with different characteristics, as observed in the mouse. Thus, our newly identified markers, Tie2 and GD2, in combination with CD24, defined the irreversible cellular hierarchy in the mouse and human NP (Fig. 7).

Among the populations of hNP cells sorted with these markers, $\mathrm{T} / \mathrm{sp}$ and TG/dp cells were conspicuous by their expression of CD271. However, because of the limited proliferative ability of single T/sp cells, TG/dp cells were used for clonal experiments to verify their stem cell properties. As expected, when transplanted in vivo, TG/dp reorganized NP tissue and showed a high ECMproducing ability. In our previous study of xenografts using normal NP tissue from Lewis rats, implanted cells disappeared by 3 weeks ${ }^{28}$. This evidence demonstrates an immune response to subcutaneous xenografts even in the NOD/SCID mouse, and, accordingly, confirms the efficacy of TG/dp cells. The T/sp cells are currently the most primitive that have been identified and, because of their low proliferative ability, appear to be dormant (Supplementary Fig. S4), although Tie2/Ang-1 signalling encourages these $\mathrm{T} / \mathrm{sp}$ cells to proliferate in vitro. Therefore, $\mathrm{T} / \mathrm{sp}$ cells might be identified if they could be expanded sufficiently without losing their properties.

In clinical IVD specimens, more than $50 \%$ of the living cells from donors aged 18-21 years expressed Tie2, suggesting a large pool of progenitors. However, only a small proportion of these cells may be progenitor cells, and their clonal multipotency towards mesenchymal lineages was heterogeneous. Therefore, markers that are more restricted are needed to distinguish NP stem cells. Alternatively, these observations may be attributable to the quality of the NP tissues obtained from surgical specimens. Our specimens were not from healthy people, and the data should be interpreted carefully in association with the Thompson disc degeneration grading ${ }^{29}$ and the Pfirrmann classification ${ }^{30}$.

Surgically removed human NP tissues are mainly semi-fibrous except for gelatinous NP tissues from younger donors. In spite of the AF regions of the samples are more fibrous and show more lamellar formation than NP, the boundary between the NP and $\mathrm{AF}$ is obscure. To address this issue, we examined healthy mature mouse IVD tissues. In the mouse, the AF was well defined and could be protected from visible contamination to the NP in the samples collected for the in vitro experiments. Our immunohistochemical analysis showed the expression of the set of marker proteins in both the mouse and human NP. As in the mature mouse disc there is clear demarcation between AF and NP tissues, our results confirm the observation that progenitor cells are 


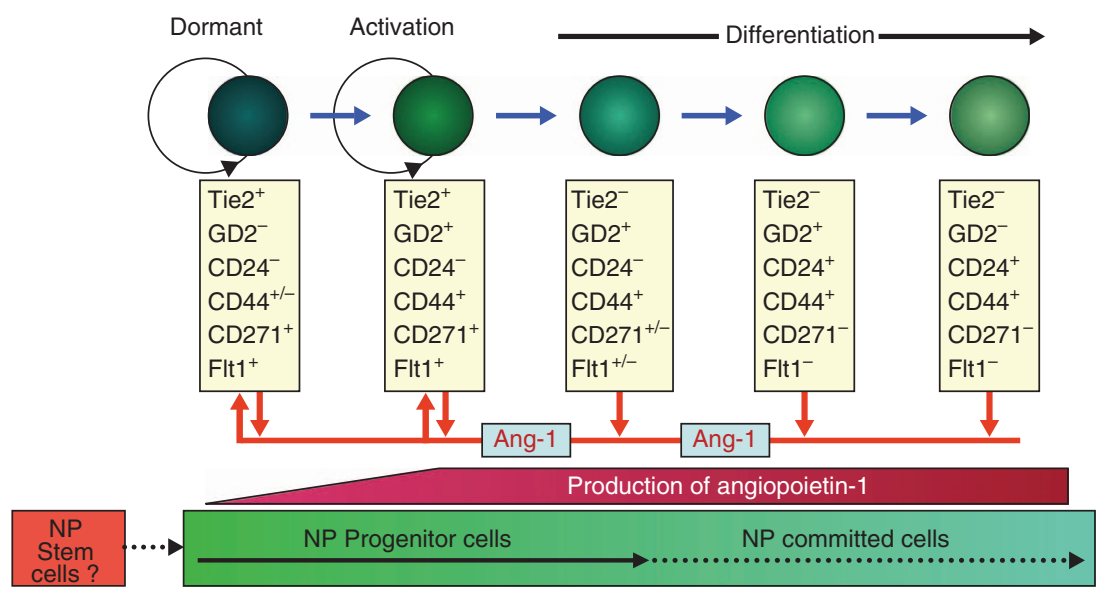

Figure 7 | Schematic model of NP cell differentiation. NP cell differentiation pathway was identified by cell surface markers based on experimental results. Individual surface marker expression is shown based on the experimental results (Supplementary Fig. S6). The arrowed circle indicates self-renewal; the blue arrows indicate irreversible change; the red arrows indicate supply of Ang-1 protein.

present in NP tissues. Both the flow cytometry and the histological analysis showed the presence of the same progenitor markers in hNP tissue as those found in the mNP. This is of interest because the cell population within the disc, especially in the NP, is reported to differ between species ${ }^{31}$.

Our study shows for the first time an experimental model of NP differentiation induced from functional NP progenitor cells in vivo. Although further study is needed to elucidate the significance of Tie2 expression by NP cells, our data suggest that Tie2 is a sensitive marker of ageing and degeneration of IVDs, and will be a useful marker for the diagnosis of IVD degeneration. The effect of Tie2/Ang-1 signalling suggests that it has a niche function that could be a target for cytokine or gene therapy. Of the various biological therapies being tested, investigations of cell therapy have shown promising results in IVD degeneration treatment in animals ${ }^{32,33}$ and have been extended recently to humans ${ }^{34,35}$. In subsequent iterations of such trials, we will test the ability of NP progenitor cells to induce tissue regeneration by delivering their resilient ECM-producing descendants. In addition, identifying the differentiation pathway of NP lineage cells will provide fundamental information for understanding IVD biology and informing therapeutic strategies.

\section{Methods}

Mice. Male C57BL/6 mice (CLEA Japan) were used for cell preparation, and male NOD/SCID mice (CLEA Japan) were used for in vivo transplantation. The animal experiments were approved by the Tokai University and were in accordance with the guidelines for the care and use of animals for scientific purposes at the Tokai University.

Mouse NP tissues. NP tissues from mouse tail IVDs were collected using a surgical microscope.

hNP tissue specimens. This study was approved by the Institutional Ethics Review Board of Tokai University School of Medicine. We obtained informed consent from the patients for the use of their IVD tissues. Twenty-three patients undergoing surgery for a burst fracture $(n=6)$, lumbar disc herniation $(n=14)$ or spondylolysis $(n=3)$ were enrolled in this study. The age of the patients ranged from 18 to 69 years, and tissue was evaluated with the Thompson grading system ${ }^{29}$ and Pfirmann classification system ${ }^{30}$.

In cases of burst fracture where the upper end-plate was injured with a fractured vertebral body and the bottom end-plate was intact, we harvested the NP tissue from the disc attached to the intact bottom end-plate. In cases of grade III-IV discs, it was difficult to distinguish between the NP and inner AF, so we harvested the tissue from the central region of the NP.

Cell isolation and culture. hNP and mNP tissues were digested with TrypLE Express (Gibco) and then collagenase-P (Roche), washed twice with $\alpha$-minimal essential medium ( $\alpha$-MEM; Gibco) and cultured in $\alpha$-MEM supplemented with $10 \%$ fetal bovine serum (Cell Culture Bioscience). All cell culture was performed under hypoxic conditions of $5 \% \mathrm{O}_{2}$ and $5 \% \mathrm{CO}_{2}$ at $37^{\circ} \mathrm{C}$.

The in vitro multidifferentiation potential of purified human NP cells was studied as described in Supplementary Methods.

Colony-forming assay. To assess colony formation, a single-cell suspension of $1.0 \times 10^{3} \mathrm{hNP}$ or mNP cells was seeded into $35-\mathrm{mm}$ diameter dishes and cultured in $1 \mathrm{ml}$ of MethoCult H4230 methylcellulose medium (Stem Cell Technologies) for 10 days. Colonies ( $>10$ cells) were counted using an inverted microscope.

Identification of a marker to select colony-forming cells. During the culture of primary $\mathrm{mNP}$ and hNP cells, $3 \times 10^{3}$ cells were collected every 3 days and subjected to the CFA. The frequency of spherical colonies was recorded. At the same time, changes in the expression of various cell surface markers on the cells were analysed by flow cytometry. Any marker that changed during the culture was recorded.

Flow cytometry and cell sorting. Cells were analysed using a FACSCalibur flow cytometer (BD Biosciences) and sorted using a FACS Vantage cell sorter (BD Biosciences). Only living cells were targeted by using a living (PI negative) gate except in the apoptosis assay. Cell cloning was also analysed using the FACSVantage cell sorter. The monoclonal antibodies used for analysis and purification are listed in Supplementary Methods.

Histochemistry and immunohistochemistry. Samples from human and mouse tissues and pellet cultures were fixed with $4 \%$ paraformaldehyde in phosphate buffer for $24 \mathrm{~h}$. Frozen or paraffin sections were cut and stained as described in Supplementary Methods. Images were captured using an LSM510 12 META confocal microscope (Carl Zeiss) or an IX70 phase microscope (Olympus).

In vivo transplantation. One hundred thousand sorted and EGFP gene-transduced $\mathrm{hNP}$ cells were suspended in phosphate-buffered saline $(100 \mu \mathrm{l})$ and injected subcutaneously into the flank area of NOD/SCID mice together with 15-Gy-irradiated 0.10 -g hNP tissue (from a 21-year-old male donor not listed in Supplementary Table S2). Eight weeks after transplantation, the mice were killed under ether anaesthesia, and the subcutaneous human NP cell cysts were excised.

Multipotent differentiation analysis in vivo. One hundred thousand sorted and EGFP gene-transduced human NP cells were transplanted into injured tail IVDs and bone defects in mice as described in Supplementary Methods.

Apoptosis assay. To identify apoptosis in hNP cells, $10 \mu \mathrm{g} \mathrm{ml}^{-1}$ goat anti-Tie2blocking polyclonal antibody (R\&D Systems) or goat control Ig (R\&D Systems) was added to the serum-free culture medium. After $48 \mathrm{~h}$, the cells were stained with $\mathrm{PI}$ and fluorescein isothiocyanate-conjugated Annexin- $\mathrm{V}$, according to the manufacturer's instructions (Beckman Coulter).

Real-time RT-PCR. Cells were homogenized in lysis buffer, and total RNA was prepared using the RNAqueous Micro RNA isolation kit (Ambion, Applied 
Biosystems). mRNA was reverse transcribed using the High Capacity RNA-tocDNA kit (Applied Biosystems). PCR analysis of the gene target was performed as described previously ${ }^{36}$. TaqMan gene expression assays (Applied Biosystems) were used as primers and probes.

Statistical analysis. Data are presented as the mean \pm s.d. Statistical significance was assessed by analysis of variance (ANOVA), and the Mann-Whitney $U$-test was used as a post hoc test. Significance was set as ${ }^{\star} P<0.05$ and ${ }^{*} P<0.01$ unless otherwise indicated. Correlations were analysed using the Spearman correlation coefficient.

\section{References}

1. Freemont, A. J. The cellular pathobiology of the degenerate intervertebral disc and discogenic back pain. Rheumatology 48, 5-10 (2009).

2. Sakai, D. Future perspectives of cell-based therapy for intervertebral disease. Eur. Spine J. 17(Suppl 4): 452-458 (2008).

3. Urban, J. P. \& Roberts, S. Degeneration of the intervertebral disc. Arthritis Res. Ther. 5, 120-130 (2003)

4. Antoniou, J. et al. The human lumbar intervertebral disc: evidence for changes in the biosynthesis and denaturation of the extracellular matrix with growth, maturation, ageing, and degeneration. J. Clin. Invest. 98, 996-1003 (1998).

5. Geiger, H. \& Van Zant, G. The aging of lympho-hematopoietic stem cells. Nat. Immunol. 3, 329-333 (2002).

6. Roberts, S., Evans, H., Trivedi, J. \& Menage, J. Histology and pathology of the human intervertebral disc. J. Bone Joint Surg. Am. 88(Suppl 2): 10-14 (2006).

7. Donohue, P. J., Jahnke, M. R., Blaha, J. D. \& Caterson, B. Characterization of link protein(s) from human intervertebral-disc tissues. Biochem. J. 251, 739-747 (1998).

8. Roberts, S., Evans, E. H., Kletsas, D., Jaffray, D. C. \& Eisenstein, S. M. Senescence in human intervertebral discs. Eur. Spine J. 15(Suppl 3): S312-S316 (2006).

9. Blanco, J. F. et al. Isolation and characterization of mesenchymal stromal cells from human degenerated nucleus pulposus: comparison with bone marrow mesenchymal stromal cells from the same subjects. Spine 35, 2259-2265 (2010).

10. Feng, G. et al. Multipotential differentiation of human annulus fibrosus cells: an in vitro study. J. Bone Joint Surg. Am. 92, 675-685 (2010).

11. Risbud, M. V. et al. Evidence for skeletal progenitor cells in the degenerate human intervertebral disc. Spine 32, 2537-2544 (2007).

12. Nakahata, T. \& Ogawa, M. Clonal origin of murine hemopoietic colonies with apparent restriction to granulocyte-macrophage-megakaryocyte (GMM) differentiation. J. Cell Physiol. 111, 239-246 (1982).

13. Benndorf, R. A. et al. Mobilization of putative high-proliferative-potential endothelial colony-forming cells during antihypertensive treatment in patients with essential hypertension. Stem Cells Dev. 16, 329-338 (2007).

14. Kawase, Y., Yanagi, Y., Takato, T., Fujimoto, M. \& Okochi, H. Characterization of multipotent adult stem cells from the skin: transforming growth factor- $\beta$ (TGF- $\beta$ ) facilitates cell growth. Exp. Cell Res. 295, 194-203 (2004).

15. Kobayashi, T. et al. Formation of mast cell colonies in methylcellulose by mouse peritoneal cells and differentiation of these cloned cells in both the skin and the gastric mucosa of $\mathrm{W} / \mathrm{Wv}$ mice: evidence that a common precursor can give rise to both 'connective tissue-type' and 'mucosal' mast cells. J. Immunol. 136, 1378-1384 (1986).

16. Martinez, C., Hofmann, T. J., Marino, R., Dominici, M. \& Horwitz, E. M. Human bone marrow mesenchymal stromal cells express the neural ganglioside GD2: a novel surface marker for the identification of MSCs. Blood 109, 4245-4248 (2007).

17. Xu, J. et al. Neural ganglioside GD2 identifies a subpopulation of mesenchymal stem cells in umbilical cord. Cell. Physiol. Biochem. 23, 415-424 (2009).

18. Jones, E. A. et al. Isolation and characterization of bone marrow multipotential mesenchymal progenitor cells. Arthritis Rheum. 46, 3349-3360 (2002).

19. Buhring, H. J. et al. Novel markers for the prospective isolation of human MSC. Ann. N Y Acad. Sci. 1106, 262-271 (2007).

20. Quirici, N. et al. Anti-L-NGFR and -CD34 monoclonal antibodies identify multipotent mesenchymal stem cells in human adipose tissue. Stem Cells Dev. 19, 915-925 (2010)

21. Hermida-Gomez, T. et al. Bone marrow cells immunomagnetically selected for $\mathrm{CD} 271^{+}$antigen promote in vitro the repair of articular cartilage defects. Tissue Eng. Part A 17, 1169-1179 (2011).

22. Nakamura, Y. et al. Angiopoietin-1 supports induction of hematopoietic activity in human CD34- bone marrow cells. Exp. Hematol. 35, 1872-1883 (2007).

23. Inoue, H., Kondo, J., Koike, T., Shukunami, C. \& Hiraki, Y. Identification of an autocrine chondrocyte colony-stimulating factor: chondromodulin-I stimulates the colony formation of growth plate chondrocytes in agarose culture. Biochem. Biophys. Res. Commun. 241, 395-400 (1997).

24. Arai, F. et al. Tie2/angiopoietin-1 signaling regulates hematopoietic stem cell quiescence in the bone marrow niche. Cell 118, 149-161 (2004).

25. Fujita, N. et al. Vascular endothelial growth factor-A is a survival factor for nucleus pulposus cells in the intervertebral disc. Biochem. Biophys. Res. Commun. 372, 367-372 (2008)

26. Oh, H. et al. Hypoxia and vascular endothelial growth factor selectively upregulate angiopoietin-2 in bovine microvascular endothelial cells. J Biol. Chem 274, 15732-15739 (1999).

27. Fujita, N. et al. CD24 is expressed specifically in the nucleus pulposus of intervertebral discs. Biochem. Biophys. Res. Commun. 338, 1890-1896 (2005).

28. Murai, K. et al. Primary immune system responders to nucleus pulposus cells: evidence for immune response in disc herniation. Eur. Cell Mater. 19, 13-21 (2010).

29. Thompson, J. P. et al. Preliminary evaluation of a scheme for grading the gross morphology of the human intervertebral disc. Spine 15, 411-415 (1990).

30. Pfirrmann, C. W., Metzdorf, A., Zanetti, M., Hodler, J. \& Boos, N. Magnetic resonance classification of lumbar intervertebral disc degeneration. Spine 26, 1873-1878 (2001).

31. Henriksson, H. et al. Identification of cell proliferation zones, progenitor cells and a potential stem cell niche in the intervertebral disc region: a study in four species. Spine 34, 2278-2287 (2009).

32. Hiyama, A. et al. Transplantation of mesenchymal stem cells in a canine disc degeneration model. J. Orthop. Res. 26, 589-600 (2008).

33. Sakai, D. et al. Differentiation of mesenchymal stem cells transplanted to a rabbit degenerative disc model: potential and limitations for stem cell therapy in disc regeneration. Spine 30, 2379-2387 (2005).

34. Watanabe, T. et al. Human nucleus pulposus cells significantly enhanced biological properties in a coculture system with direct cell-to-cell contact with autologous mesenchymal stem cells. J. Orthop. Res. 28, 623-631 (2010).

35. Yoshikawa, T., Ueda, Y., Miyazaki, K., Koizumi, M. \& Takakura, Y. Disc regeneration therapy using marrow mesenchymal cell transplantation: a report of two case studies. Spine 35, E475-E480 (2010).

36. Sakai, D., Nakai, T., Mochida, J., Alini, M. \& Grad, S. Differential phenotype of intervertebral disc cells: microarray and immunohistochemical analysis of canine nucleus pulposus and annulus fibrosus. Spine 34, 1448-1456 (2009).

\section{Acknowledgements}

We are grateful to staff of the Education and Research Support Center, Tokai University, Tomoko Uno and Kunihiko Murai for excellent assistance and advice. This work was supported in part by a Grant-in-Aid for Scientific Research (20791056) from the Ministry of Education, Culture, Sports, Science and Technology of Japan; a grant from AO Spine International (AOSBRC-07-1) to D.S.; and a Research Grant to K.A. from The Science Frontier Program of the Ministry of Education, Culture, Sports, Science and Technology of Japan. This work was also supported by Research Grants on Regenerative Medicine for Clinical Application (H20-Saisei-Ippan-006 to S.K. and H21-Saisei-Ippan-003 to J.M.) from the Ministry of Health, Labour and Welfare of Japan.

\section{Author contributions}

D.S., Y.N. and T.N. contributed to the study design, performed experiments and wrote the manuscript. T.M. performed experimental work. K.A., S.G., M.R., D.C. and M.A helped with the organization of the study. K.C., K.M., S.K., K.Y., H.O. and J.M. contributed to the experimental design and manuscript editing.

\section{Additional information}

Supplementary Information accompanies this paper at http://www.nature.com/ naturecommunication

Competing financial interests: The authors declare no competing financial interests

Reprints and permission information is available online at http://npg.nature.com/ reprintsandpermissions/

How to cite this article: Sakai, D. et al. Exhaustion of nucleus pulposus progenitor cells with ageing and degeneration of the intervertebral disc. Nat. Commun. 3:1264 doi: $10.1038 /$ ncomms2226 (2012).

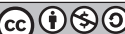

This work is licensed under a Creative Commons AttributionNonCommercial-ShareAlike 3.0 Unported License. To view a copy of this license, visit http://creativecommons.org/licenses/by-nc-sa/3.0/ 